\title{
La "tierra irrigada" y la mercantilización del agua en un nuevo paisaje hídrico de la agricultura pampeana: el caso del clúster de la semilla
}

\author{
"Irrigated land" and the commodification of water in a \\ new waterscape of Pampean agriculture: the case of the \\ seed cluster
}

'Doctora en Antropología. Investigadora, Instituto de Geografía "Romualdo Ardissone", Facultad de Filosofía y Letras, Universidad de Buenos Aires, Investigadora Asistente, Consejo Nacional de Investigaciones Científicas y Técnicas, Buenos Aires, Argentina. $\square$ (iD)
RESUMEN Desde mediados de la década de 1990 la agricultura extensiva bajo riego creció aceleradamente en la Argentina. Donde tradicionalmente se producía en secano, la adopción de tecnología creó un nuevo paisaje hídrico: la "tierra irrigada". Para comprender la creación de dicha categoría conceptual y su anclaje dentro de un modelo productivo específico, en este trabajo me propongo analizar la difusión del riego mecanizado en el norte de la provincia de Buenos Aires a partir de las principales políticas públicas que intervienen en esta transformación territorial: el Plan de Mejora Competitiva del clúster de la semilla, el Plan Nacional de Riego y la gestión del agua subterránea para el riego productivo. Recuperando aportes de la ecología política y de los estudios de la ciencia y la tecnología, argumento que la explotación del agua subterránea para riego implica una privatización del recurso que permanece invisibilizada. La "tierra irrigada" contiene dos de los recursos fundamentales para la producción de alimentos que, asociados, se resignifican. El agua adquiere sentido productivo como una mercancía a la que es factible asignar un valor monetario y se privatiza de hecho en el momento de su consumo productivo; mientras la tierra, gracias al agua, se valoriza económicamente al permitir nuevas alternativas de explotación con mayores márgenes de rentabilidad. Así, el agua es el "lubricante" que refuerza el proceso de acumulación.

PALABRAS CLAVES Agua Subterránea; Riego Agrícola; Política Pública; Argentina.

ABSTRACT Since the mid-1990s extensive agriculture using irrigation has grown rapidly in Argentina. Where farming on dry land was traditionally carried out, technological change has created a new waterscape: "irrigated land." To understand the creation of this conceptual category and how it is anchored in a specific production model, in this paper I contextualize the spread of mechanized irrigation in the north of the province of Buenos Aires by examining the main public policies that intervene in this territorial transformation: the Competitive Improvement Plan of the Seed Cluster, the National Irrigation Plan, and the management of groundwater for productive irrigation. Integrating contributions from Political Ecology and Science and Technology Studies, I argue that the exploitation of groundwater for irrigation implies the privatization of a resource that remains invisible. "Irrigated land" contains two of the fundamental resources for food production that, jointly, resignify each other. Water acquires productive meaning as a commodity to which it is feasible to assign a monetary value, and becomes privatized de facto at the moment of its productive consumption; while the land - thanks to water - is valorized economically by allowing for new exploitation alternatives with higher profit margins. Thus, water acts as the "lubricant" that reinforces the accumulation process.

KEY WORDS Groundwater; Agricultural Irrigation; Public Policy; Argentina. 


\section{INTRODUCCIÓN}

En las últimas décadas se produjo un acelerado crecimiento de la agricultura irrigada en varios países del mundo. Posibilitado por la tecnología de perforación y riego que surgió a partir de 1970 y facilitó el acceso al agua subterránea ${ }^{(1,2)}$, este desarrollo estuvo comandado en gran medida por empresarios agrícolas de mediana y gran escala dedicados a la agricultura industrial de productos de exportación, en especial, en el continente américano ${ }^{(3,4,5)}$.

En el marco del capitalismo global, este proceso implica el desarrollo de formas de mercantilización del agua que no necesariamente conllevan la creación de mercados formales. La mercantilización radica en que el acceso y uso del agua dependen más o menos directamente de la capacidad de compra de sus usuarios. En el riego agrícola, el agua subterránea es para quien pueda realizar la inversión en la tecnología necesaria. En el caso de la provisión de agua para consumo humano, su acceso depende de que los usuarios puedan pagar las tarifas a las empresas proveedoras del servicio ${ }^{(6)}$. En ambas situaciones, el agua, un bien común, asume un carácter privado de facto.

Esta nueva forma de mercantilización del agua puede entenderse como parte de la dinámica que O'Connor denominó en 1996 "la segunda contradicción del capital"(7). Según O'Connor, además de la contradicción entre capital y trabajo, el sistema capitalista opera sobre la base de una segunda contradicción que existe entre naturaleza y sociedad. Dada la apropiación material cada vez mayor de los recursos que se realiza en la producción capitalista, y la capacidad limitada del ambiente de, por un lado, proporcionar el material necesario para las actividades productivas $y$, por el otro, absorber los desechos generados como subproductos del proceso productivo -como la contaminación- se suscita una contradicción en la que se destruyen las condiciones de producción. En términos de desarrollo agrícola, dicha contradicción explica que, si bien el riego puede ser sinónimo de crecimiento económico, también puede significar deterioro ambiental, especialmente en el marco de un pobre andamiaje institucional y escaso conocimiento ${ }^{(8)}$, produciendo el agotamiento y contaminación de las fuentes de agua subterránea ${ }^{(9)}$.

En esta línea de análisis y siguiendo a Harvey ${ }^{(10)}$, son hechos que corresponden a un proceso general de mercantilización de la naturaleza y de acumulación por despojo, intrínseco al sistema capitalista. Este autor recupera el concepto de acumulación originaria ${ }^{(11)}$ y discute que haya existido un proceso de expropiación histórico, "primitivo", que sucedió una única vez en el origen del capitalismo. Al contrario, según Harvey la acumulación actúa permanentemente sobre los bienes comunes y, entre ellos, los recursos hídricos, que dejan de ser considerados patrimonio común al ser convertidos en mercancías que redundan en crecimiento económico para quien los posee ${ }^{(12)}$. Por la finitud de los recursos y la rivalidad que su usufructo implica ${ }^{(13)}$, la apropiación individual abre el camino a la concentración en forma de derechos de uso con la consiguiente expropiación de quienes quedan excluidos del acceso al agua. Esta dinámica permite al mismo tiempo la deslocalización material de los recursos y de sus usuarios, a través de acueductos y canales o incorporados en mercancías, como los granos.

Al igual que en la acumulación originaria, en la acumulación por despojo, el Estado juega un rol crucial en respaldar y promover estos procesos ${ }^{(10)}$. Bajo el neoliberalismo, lejos de reducirse el poder estatal a un rol pasivo de "dejar hacer", el Estado adopta una posición activa mediante políticas públicas funcionales a la actividad del capital privado reforzando la mercantilización.

En el norte de la provincia de Buenos Aires, Argentina, la agricultura irrigada surgió y prosperó por fuera de las iniciativas estatales, lejos de la regulación y el orden del poder público. En este trabajo presento tres políticas públicas que tienen el efecto de consolidar el espacio irrigado, encauzando las dinámicas sociales y económicas que estaban operando. Para ello, estas políticas procuran asignar al Estado un nuevo rol, que no sea el de mero 
espectador que avala el modo de producción vigente desde la "lógica de la omisión". Susann Baez Ullberg describe esta lógica, en referencia a la acción de los agentes gubernamentales en la construcción de riesgos, como "inherente al mundo político argentino, fuertemente marcado por las relaciones clientelares y una burocracia politizada, a las formas existentes de legislación, a la forma en que se toman decisiones políticas y a la manera en que estas se implementan, se comunican y son documentadas por la burocracia argentina"(14). Así las políticas revisadas implican una formulación explicita de un modelo de desarrollo que puede observase en los objetivos que se proponen.

Las políticas públicas pueden ser definidas como el "conjunto de acciones y omisiones que manifiestan una determinada modalidad de intervención del Estado en relación con una cuestión que concita la atención, el interés o la movilización de otros actores en la sociedad civil"(15). Sin embargo, desde una perspectiva antropológica, el concepto de políticas públicas no tiene una definición acabada, sino que se considera que adquirirá su sentido dentro de cada contexto particular. Aun teniendo en cuenta las particularidades de cada caso, "las políticas reflejan maneras de pensar sobre el mundo y cómo actuar en él. Contienen modelos implícitos -y algunas veces explícitos- de una sociedad y de visiones de cómo los individuos deben relacionarse con la sociedad y los unos con los otros" ${ }^{\prime \prime(16)}$. Sobre la base de este modelo, y de un modo instrumental, operan como herramientas de intervención y acción social para administrar, regular y cambiar la sociedad. En este sentido, las políticas públicas están interesadas en imponer orden y coherencia en el mundo(17).

En el caso de las políticas vinculadas al agua, en primer lugar, conviene detenerse en revisar algunos conceptos generales sobre dicho objeto. Recientemente, un abundante corpus de bibliografía en antropología y geografía plantea la necesidad de entender este elemento como una entidad de carácter híbrido, al mismo tiempo natural y cultural, que fluye junto con el poder en un ciclo hidrosocial ${ }^{(18,19,20,21)}$. Ello permite analizar desde la ecología política la multiplicidad de actores involucrados y los distintos valores en juego que participan del conjunto de relaciones de apropiación, control, resistencia y disputa $^{(22,23,24,25)}$. Estas relaciones van configurando paisajes hídricos que expresan la organización social y las estructuras institucionales y normativas que dan forma a la gobernanza del agua ${ }^{(26)}$.

A pesar de las diferencias que pueden señalarse entre las tres políticas presentadas a continuación, se trata de medidas que legitiman y promueven la cesión de lo público, el recurso común, a la explotación y control privado, autorizando prácticas irregulares que venían operando de manera tácita. Ello constituye una privatización de hecho del agua, de la que se benefician en gran parte compañías transnacionales y a la cual estas políticas intentan dar un formato institucional mediante "asociaciones civiles", "corporaciones" y "consorcios". De hecho, la institucionalización es parte central de dichas políticas, en tanto buscan aportar una arquitectura organizacional consistente con el proceso productivo.

Este tipo de procesos de expropiación del agua subterránea con el apoyo del Estado a través de políticas de acción u omisión no son exclusivos de Argentina. En la actualidad, en la mayoría de los países, el agua subterránea es considerada un recurso público de propiedad estatal, pero dadas las dificultades que encuentran los gobiernos en implementar una regulación efectiva, se ha usado y aún se usa como si fuera de acceso abierto ${ }^{(9)}$. Ello da lugar a procesos de privatización de hecho, similares al de Argentina, en países como Brasil, México o España ${ }^{(27,28,29)}$.

La excepción paradigmática a estos regímenes de propiedad públicos del agua es Chile. Allí el agua es administrada por el mercado con base en una asignación de derechos privados que sus propietarios pueden comprar y vender libremente. Este mecanismo, lejos de zanjar el proceso de acumulación, lo complejiza, aumentando la concentración e inequidad en la distribución del recurso, poniendo en riesgo la sustentabilidad de los 
sistemas hídricos y el derecho humano de acceso al agua ${ }^{(30,31)}$. En EEUU, cada estado tiene un marco regulatorio particular para el agua subterránea ${ }^{(4)}$, siempre sobre la base de derechos de uso de propiedad privada, mostrando una herterogeneidad importante en las doctrinas de asignación de derechos y sus formas de manejo ${ }^{(9,32)}$. Estos derechos de uso no implican necesariamente propiedad del agua, que puede quedar reservada a los estados. Por eso, los derechos de agua que no sean utilizados en determinados períodos pueden ser reasignados por los estados, como ha sucedido en Kansas ${ }^{(33)}$.

En otros lugares del mundo, la mercantilización del agua subterránea para riego adquiere formas institucionales que, aunque informales y no reguladas, permiten la comercialización de volúmenes de agua. Este es un fenómeno relativamente reciente en China ${ }^{(34)}$. En la región norte, pequeños agricultores venden y compran agua en un mercado informal autoorganizado, donde el Estado ha cumplido un rol importante dentro de este desarrollo, facilitando el acceso al capital para las inversiones en riego y omitiendo controles locales. Del mismo modo, se han generado mercados localizados en el sur de Asia, en India, Pakistán y Bangladesh, donde en las últimas décadas se ha observado un crecimiento explosivo de la agricultura bajo riego sobre la base del agua subterránea. Como consecuencia, una región que tenía una gran tradición de riego de manejo comunitario o Estatal, se tornó progresivamente anárquica, gracias a que la nueva agricultura bajo riego se sustenta en el uso no regulado de pozos de riego privados ${ }^{(8,26)}$.

Dentro de la mercantilización global del agua, muchas veces son actores transnacionales los que están involucrados, profundizando los procesos de deslocalización de los recursos. Por ejemplo, para el caso de Australia, Strang ${ }^{(35)}$ advierte que la transferencia legal o de hecho de la propiedad y control del agua a manos privadas es facilitada por mecanismos regulatorios débiles, que implican una transferencia de activos y poder a empresas transnacionales, en desmedro de las poblaciones locales.
En consonancia con estos procesos observados a nivel mundial, desde mediados de la década de 1990, en Argentina hubo un creciente desarrollo de la agricultura extensiva bajo riego con uso de agua subterránea, que implicó una mercantilización del agua. Las provincias con mayor superficie irrigada fueron Buenos Aires y Córdoba, lo que indica el carácter pampeano de esta tecnología. En la provincia de Buenos Aires, según estimaciones del Programa Nacional de Agua del Instituto Nacional de Tecnología Agropecuaria (INTA), entre el año 2000 y 2015, la superficie bajo riego por sistema de aspersión y pivote central creció a una tasa anual de más del $18 \%$. Este crecimiento se produjo gracias a que muchos agricultores incorporaron de forma privada sistemas de riego mecanizado para el cultivo de maíz, trigo, soja o papa, utilizando agua subterránea que bombean de acuíferos profundos. En el caso del norte de la provincia, el riego se utiliza para la producción de semillas de maíz híbrido para empresas transnacionales, principalmente.

Con la adopción del riego mecanizado se produjo una transformación del espacio rural pampeano. Donde tradicionalmente se producía en secano, surge la "tierra irrigada" como una nueva categoría conceptual de espacio agrario; un objeto que, al mismo tiempo, tiene efectos que actúan sobre otros -actores y objetos- para crear este nuevo paisaje. Para entender su creación y anclaje dentro de un modelo productivo específico, me propongo analizar la acción del Estado, vinculando el proceso de difusión del riego mecanizado en la zona núcleo de producción de granos en el norte de la provincia de Buenos Aires con ciertas políticas públicas. Puntualmente, me refiero al Plan de Mejora Competitiva, que fomenta el desarrollo del clúster de la semilla en dicha zona, al Plan Nacional de Riego y a la política de gestión del agua subterránea para riego productivo.

A partir de este análisis, argumento que la explotación del agua subterránea para riego de semillas implica una apropiación del recurso, al que se accede según la capacidad de económica de los agricultores para realizar la inversión en el sistema de riego y según la 
necesidad de producción de las empresas semilleras que presionan por la ampliación de la superficie irrigada. Así, la "tierra irrigada" contiene dos de los recursos fundamentales para la producción de alimentos: la tierra y el agua, el primero como propiedad privada y, el segundo, como propiedad estatal, es decir, público, que aunados se resignifican mutuamente. Como se verá, el agua adquiere sentido productivo como una mercancía a la que es factible asignar un valor monetario y se privatiza de hecho en el momento de su consumo productivo mientras, gracias al agua, la tierra se valoriza económicamente al permitir nuevas alternativas de explotación con mayores márgenes de rentabilidad. Así, el agua es el "lubricante" que refuerza el proceso de acumulación ${ }^{(36)}$.

El caso del norte de la provincia de Buenos Aires permite observar cómo este modo de explotación de los recursos implica la asociación de políticas públicas, actores institucionales, empresas y saberes expertos que profundizan la segunda contradicción del capital, actualizando una novedosa forma de mercantilización del agua al generar un ensamblaje que se expresa en el surgimiento de la "tierra irrigada", como un nuevo actorred $^{(37)}$. Para ello, tomamos algunos elementos de los estudios de la ciencia y tecnología Science and Technology Studies (STS)- y de la teoría de actor-red, desarrollada desde la década de 1980 por teóricos como Bruno Latour $^{(37)}$ y Michael Callon ${ }^{(38)}$ que permite considerar la capacidad de acción de los elementos no humanos.

\section{METODOLOGÍA}

Para llevar adelante este análisis, utilicé centralmente información secundaria, en especial, resoluciones de organismos públicos, informes, y artículos periodísticos, e información primaria relevada durante sucesivos trabajos de campo realizados en la zona de estudio desde 2014. En efecto, presento aquí resultados parciales de un proyecto de investigación en curso titulado, "Moderna tecnología de riego para la producción de cultivos extensivos: las paradojas del uso agrícola del agua subterránea en la construcción social del riesgo en la cuenca del río Arrecifes". Esta investigación está orientada por una estrategia metodológica cualitativa y flexible, basada en un trabajo de campo multisituado ${ }^{(39)}$.

La flexibilidad, necesaria para un abordaje cualitativo, remite a la predisposición del investigador y trabajador de campo, a la apertura para reconocer datos y aristas de la investigación no contempladas en el diseño original. Ello permite ir (re)orientando el trabajo a medida que surgen los descubrimientos. En la lógica y proceso de la investigación cualitativa, "cada componente del diseño puede necesitar ser reconsiderado o modificado en respuesta a nuevos desarrollos o a cambios en alguno de los otros componentes $^{\prime \prime(40)}$. La flexibilidad de la investigación, por lo tanto, implica un proceso de permanente reflexividad $^{(41)}$.

Durante los trabajos de campo realicé observaciones en terreno, participé de eventos del sector y conduje distintos tipos de entrevistas. El corpus de información primaria consiste en 36 entrevistas semiestructuradas y en profundidad y notas de observaciones de campo ${ }^{(41)}$. Las entrevistas fueron pautadas con anterioridad al encuentro. Al momento de solicitar las entrevistas se les informó a los entrevistados sobre el proyecto de investigación en el marco del cual se realizarían dichas entrevistas y las condiciones de anonimato y confidencialidad que implicaba su participación.

Los entrevistados fueron seleccionados teniendo en cuenta su experiencia próxima con la agricultura irrigada de la zona ${ }^{(42)}$. Dicha experiencia radica, en primer lugar, en ser productores usuarios del riego. Coincidentemente, los regantes a los que tuve acceso de la zona integraban el Movimiento CREA. Se entrevistó al asesor del Movimiento y a los encargados de la gestión de las empresas agropecuarias que integraban los grupos (administradores de campos y productores de empresas agropecuarias familiares). En segundo lugar, se entrevistó a técnicos del 
Instituto Nacional de Tecnología Agropecuaria que han investigado aspectos agronómicos vinculados al uso del riego en la zona; a funcionarios de gobiernos locales del área de producción o que tienen a su cargo la gestión de recursos hídricos, caminos y controles ambientales; y a técnicos asesores y gestores de la Asociación de Riego Pampeano, como así también de la Asociación Argentina de Semilleros. Finalmente, se entrevistó a ingenieros agrónomos vendedores de equipos de riego o que se desempañaran como empleados en las empresas semilleras del clúster con experiencia en el área de producción de maíz híbrido.

Las entrevistas se organizaron en dos partes: una de intercambio informal -presentaciones, cierres y despedidas- y otra más estructurada, orientada por una guía de preguntas adaptada a cada tipo de actor, con las que se indagaba sobre aspectos generales de la labor desempeñada y la trayectoria personal (sea en la producción agropecuaria, en la gestión pública, en el asesoramiento agrícola, en la actividad comercial del riego, en la representación gremial, o dentro del ámbito corporativo de las empresas semilleras), conocimiento y uso de la tecnología de riego, percepción del ambiente y sus recursos, vinculación con otros actores, problemas y conflictos ambientales. Esta segunda parte fue grabada en archivos de audio que luego fueron transcriptos junto con las anotaciones de campo en registros textuales ampliados.

Sobre estos registros realicé un análisis cualitativo mediante técnicas de codificación. La estrategia de codificación se basó en la utilización de códigos ya construidos inductivamente en una investigación anterior sobre la adopción de tecnología de riego en la provincia de Córdoba, Argentina ${ }^{(5)}$. Sin embargo, las particularidades de la producción de semillas y el perfil de los actores involucrados implicaron la construcción de nuevos códigos a partir de la detección de recurrencias significativas propias del caso. En ambos casos, la técnica de codificación consistió en generar códigos simples y concretos que fueron progresivamente definidos y posteriormente agrupados en categorías de mayor nivel abstracción (familias de códigos).
Una vez ordenado y codificado el material de campo, se procedió a la elaboración de una descripción de rango medio que exprese las conexiones de sentido habilitadas por el tramado de los códigos. Los resultados preliminares del análisis interpretativo de la información primaria fueron triangulados y complementados con la información secundaria.

Con respecto a la selección del área de estudio, si bien el riego mecanizado se encuentra distribuido en todo el norte de la provincia de Buenos Aires, aquí realizo un recorte espacial enfocado al "clúster de la semilla", que abarca un área de límites no definidos entre el norte de Buenos Aires y el Sur de Santa $\mathrm{Fe}^{(43)}$. Teniendo en cuenta esta selección, presento los datos de los principales partidos del norte de la provincia de Buenos Aires, donde se desarrolla la agricultura de riego: Rojas, Pergamino, Bartolomé Mitre y Salto. Dichos partidos ocupan la parte media y baja de la cuenca del río Arrecifes (Figura 1).

\section{REGAR EN LA PAMPA HÚMEDA}

En la llamada "zona núcleo" de producción de granos de la Argentina, ubicada en el norte de la provincia de Buenos Aires y sur de Santa Fe, el riego mecanizado se utiliza casi exclusivamente para producir semillas híbridas de maíz. Dadas las características del proceso productivo de estas semillas, en la segunda mitad de la década de 1980, muchas empresas y productores asociados a ellas comenzaron a producirlas mediante riego por surco con agua subterránea. Pero desde mediados de la década de 1990, esta actividad se fue mecanizando progresivamente al incorporarse equipos de riego por aspersión y pivote central.

La producción de semillas implica un proceso organizado en tres fases. En la primera, las empresas que realizan investigación y desarrollo (I+D) se dedican al "breeading" o mejoramiento vegetal a partir del cual se obtienen los nuevos germoplasmas. El cultivo de los parentales de los híbridos comerciales 


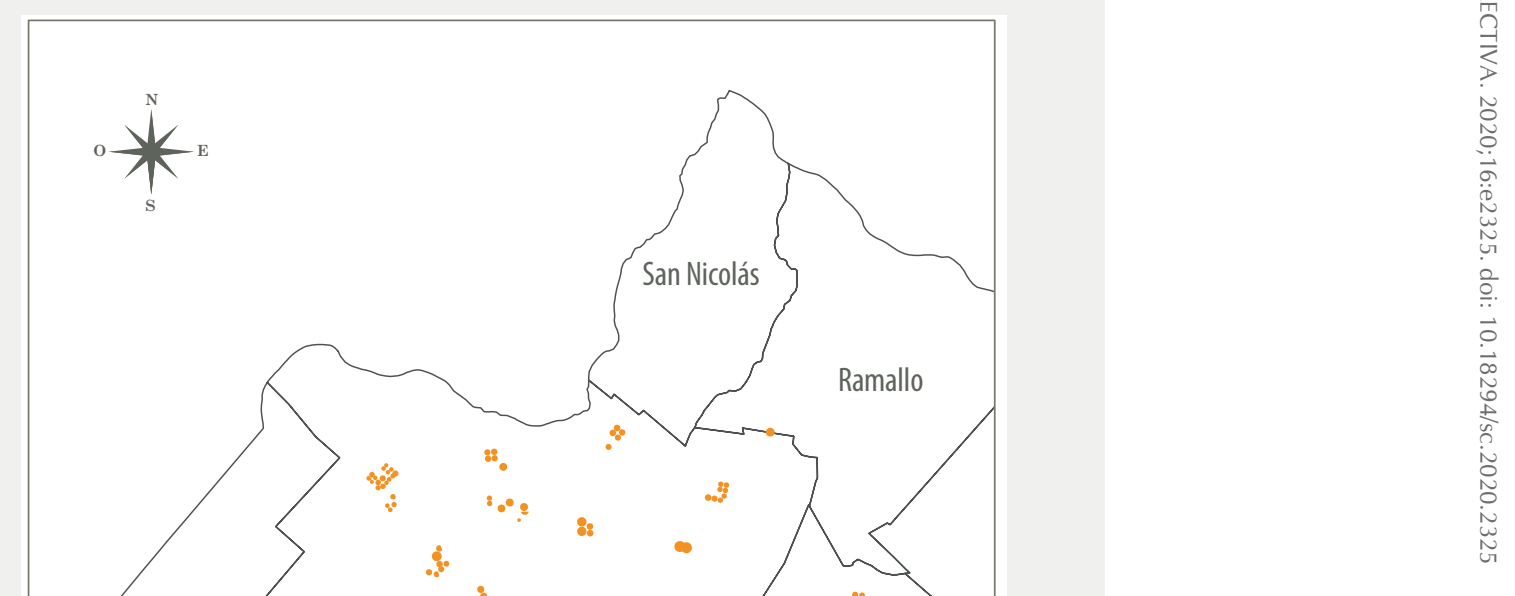

Figura 1. Distribución espacial de la superficie irrigada con equipos de pivote central en partidos del norte de la provincia de Buenos Aires, 2015.

Fuente: Elaborado por Néstor Barrionuevo para el Plan Nacional Agua, Instituto Nacional de Tecnología Agropecuaria.

es llamado "fundación" en la jerga del sector y suele realizarse en pequeñas parcelas de alrededor de $30 \mathrm{ha}$, generalmente en campos propios de las empresas (notas del trabajo de campo, noviembre 2016). La segunda fase es la "multiplicación" de dichos parentales, la cual se realiza en campos de terceros hasta un volumen adecuado para la demanda. Por último, la tercera fase de producción de semillas incluye las actividades de acondicionamiento, embolsado y comercialización del producto.

En el caso de la "tierra irrigada", la más relevante de las fases es la de multiplicación, que es donde se utiliza el riego a gran escala en la denominada "producción a campo" (notas del trabajo de campo, noviembre 2016). En ella intervienen tres actores fundamentales: 
1. Por un lado, las empresas que desarroIlan las variedades híbridas, esto es, la genética de las semillas a multiplicar. Si bien, las semillas híbridas generaron una nueva revolución verde en la cual tuvo una participación importante la I+D pública, actualmente el grueso de la oferta se encuentra concentrado en unas pocas empresas transnacionales con desarrollos biotecnológicos, y algunos semilleros locales que licencian los eventos transgénicos a las empresas desarrolladoras ${ }^{(44)}$.

2. Por otro lado, empresas tercerizadas que se dedican especialmente a la multiplicación de semillas. Es decir, las semilleras pueden encargarse ellas mismas de la multiplicación integrando todo el proceso "in house" o pueden "tercerizarlo" contratando a otras empresas que se dedican específicamente a este proceso (notas del trabajo de campo, noviembre 2016). En este sentido, "semilleros" son todas las empresas que producen semillas, aunque lo hagan como parte de un servicio a una tercera empresa o no. Esta categoría distingue claramente la forma mega empresarial de la producción, de los productores particulares, incluso en el caso de que estos últimos funcionen bajo formas jurídicas que exceden a la persona física, como las sociedades anónimas.

3. Y finalmente, los productores o dueños de los campos, que pueden alquilar la superficie a los "semilleros", o establecer un "convenio de producción" en el cual ellos mismos se convierten en socios de la empresa que hace la multiplicación.

Por lo tanto, las empresas tienen dos maneras de acceder a la tierra para la producción de semillas. La más simple es el arrendamiento, por el cual el dueño del campo da en alquiler los lotes para la producción bajo riego. Muchas veces para acceder a ese "negocio" los dueños realizan la inversión en el sistema de riego y, otras, la instalación la hacen las propias empresas semilleras que retiran los equipos una vez finalizado el contrato (notas del trabajo de campo, octubre 2017). Este arrendamiento es fijo por una cierta cantidad de años (de 2 a 5) y el productor no está vinculado a la producción. La retribución por el alquiler es fijada en quintales de soja y surge de una suerte de lucro cesante, es decir, de la rentabilidad esperada del cultivo que pudiera hacer el productor en ese lote. Si la inversión en el sistema de riego la realizó el productor o dueño del campo, el alquiler se paga entre 30 y 35 quintales de soja por ha cobrados al inicio de la campaña. De no ser así, el valor del alquiler ronda entre 14 y 15 quintales de soja por ha (notas del trabajo de campo, octubre 2017). En este último caso, la empresa se hace cargo de la incorporación del riego y debe realizar la perforación e instalar el equipo que muchas veces compran y otras veces alquilan a los mismos proveedores.

La otra opción para acceder a la tierra es el "convenio de producción", en el que el productor tiene que estar dispuesto a convertirse en "socio" de la empresa. Bajo esta modalidad, el productor se transforma en una especie de "empresa de servicios" para el semillero (notas del trabajo de campo, noviembre 2016) y debe encargarse de todas las tareas "a campo" para la multiplicación de semillas, con excepción del "despanojado" del maíz, de la cual el productor se desentiende. Como el maíz es una planta autógama, que posee en sí misma una parte masculina y otra femenina, para lograr el cruzamiento entre distintos parentales, necesario para lograr un maíz híbrido, es fundamental evitar que las partes masculinas y femeninas de la misma planta se polinicen entre sí. Por ello la producción de híbridos requiere del "despanojado". Esta es una tarea que consiste en arrancar las flores -estambres- que se localizan en la punta del maíz a las plantas que actuarán de "hembra". Esta tarea se encuentra parcialmente mecanizada, pero sigue requiriendo un aporte importante de trabajo manual de "los changos", modo en que los productores denominan a los trabajadores rurales estacionales, para que "repasen" (retiren) todas las flores que la máquina deja en el lote de cultivo (notas del trabajo de campo, mayo 2016).

Cuando la inversión en el equipo de riego y su manejo la hace el productor, además del cobro del arrendamiento, el productor suele percibir $U \$ 2$ dólares por milímetro regado por 
ha. La proliferación del convenio de producción como modalidad contractual es parte de una "tendencia hacia la tercerización" cada vez más instalada, para la multiplicación de semillas, según los entrevistados (notas del trabajo de campo, mayo 2017). El total del volumen producido -el grano "físico"- lo recupera la empresa semillera que lo acondiciona y lo embolsa para su comercialización. El productor recibe, como retribución, el dinero equivalente al rendimiento por hectárea del maíz semilla multiplicado por un factor que es negociado y fijado por contrato antes de cada campaña, según la cotización del "precio pizarra" del maíz comercial, denominación coloquial que reciben los precios de referencia pautados por los corredores que operan en el ámbito de la Bolsa de Comercio de Rosario.

\section{Políticas Públicas y transformación territorial: la consolidación de un clúster}

Si bien dentro de la economía y la geografía económica existe toda una línea de trabajo sobre los clústeres, para los objetivos aquí propuestos $\mathrm{y}$, siguiendo a Porter ${ }^{(45)}$, tomo el concepto de clúster en su acepción más general, como una concentración geográfica de empresas e instituciones interconectadas que actúan en determinado campo. En el caso del "clúster de la Semilla", se trata de 878 empresas e instituciones vinculadas a la producción de semillas (cereales, oleaginosas y forrajeras) que concentran el $95 \%$ de la actividad semiIlera nacional en un radio de $180 \mathrm{~km}$ entre las ciudades de Pergamino y Venado Tuerto ${ }^{(43)}$.

La actividad principal del clúster es la creación, desarrollo, producción y comercialización de semillas híbridas (como el maíz, el girasol y el sorgo), autógamas (como el trigo y la soja), y forrajeras (como la alfalfa, raigrás, tréboles, etc.). Estas se orientan a tres segmentos del negocio: la producción de semillas para la producción extensiva de granos, las semillas forrajeras $y$, finalmente, la producción de semillas contraestación destinadas al mercado internacional.
Dicho clúster obtuvo reconocimiento oficial en 2009, a partir de un convenio entre el Ministerio de Agricultura, Ganadería y Pesca de la Nación (MAGyP) y distintos actores que participan en él con el fin de fomentar su desarrollo. De este convenio surgió el Plan de Mejora Competitiva, la primera de las políticas aquí analizadas.

\section{El Plan de Mejora Competitiva}

El Plan de Mejora Competitiva es una política específica para el desarrollo de la industria semillera que integra el clúster. Formulado en 2011 sobre la base de una metodología participativa, este Plan parte de un análisis del sector para identificar debilidades y desarrollar estrategias que permitan aumentar su competitividad. Para ello, reconoce en primer lugar a los "principales referentes". Estos son el Instituto Nacional de Tecnología Agropecuaria (INTA) -que cuenta con una Estación Experimental en Pergamino-, el Instituto Nacional de la Semilla (INASE), la Asociación de Semilleros Argentinos (ASA), el Ministerio de Asuntos Agrarios de la provincia de Buenos Aires, el Ministerio de Producción de la provincia de Santa Fe, los municipios de Venado Tuerto y Pergamino y la Universidad Nacional del Noroeste de Buenos Aires (UNNOBA). Ello revela el mapa de actores con poder económico, técnico y gubernamental, que operan en el territorio dentro del universo social que se articula alrededor de la producción de semillas.

Dichos actores consensuaron una serie de acciones para "iniciar un proceso de clusterización regional", el cual aparece formulado como una política pública por el Programa de Servicios Agrícolas Provinciales (PROSAP) del Ministerio de Agricultura, Ganadería y Pesca, en 2013, cuando se publican los primeros resultados del diagnóstico de la "situación competitiva" del clúster. Este diagnóstico identifica cuatro grandes tendencias globales y sus consecuencias sobre el clúster:

1. Demanda global creciente en materias primas y alimentos que presiona sobre la industria semillera para incrementar rindes y capacidad para producir en nuevas zonas. 
2. Presión sobre el medioambiente por la intensificación de la producción, lo que demanda de la industria el desarrollo de semillas que permitan producir grandes volúmenes con menor uso de agua y agroquímicos.

3. El proceso de cambio tecnológico, en especial, en lo que refiere a biotecnología, necesaria para mantener posiciones competitivas en el mercado.

4. Reorganización de las cadenas y sistemas de producción, como la creciente concentración en unas pocas empresas transnacionales líderes que poseen la escala necesaria para realizar la inversión en investigación y desarrollo biotecnológico, y una cadena de valor mundial que hace que las distintas fases de producción puedan instalarse en diferentes localizaciones. Ello hace que exista una competencia a nivel global entre países y regiones por localizar, en sus territorios, las distintas fases, lo que dependerá de los "costos, capacidades y marcos regulatorios de cada lugar"(43). A propósito de ello, un técnico dedicado a la multiplicación de semillas comentaba:

\begin{abstract}
...el pedido del incremento depende de que fracase la siembra en EEUU hoy. Entonces ¿cuándo llega el pedido? el mes que viene, junio, julio. O sea, yo tengo un mes, a lo sumo dos meses, para decirle ok, si no dejo pasar muchos dólares. Es así. Si no lo respondo yo lo responde Chile, entonces, es una cuestión de... [...] Chile en 15 días te dio el permiso de explotación porque sabe que eso genera riqueza al país. (Técnico, Pergamino, mayo de 2017).
\end{abstract}

Argentina participa de esta cadena de valor global como usuario y comprador de licencias y como proveedor de semillas. A nivel nacional, la reorganización de los sistemas de producción involucra también al crecimiento de la producción de semillas por aumento y transformaciones en la demanda, dado que estas se destinan a producciones que se han expandido considerablemente en las últimas décadas, como la agricultura extensiva de granos y las cadenas de nutrición animal para la producción de carne y leche. Las líneas de acción que se proponen a partir de este diagnóstico son: 1) enfrentar los cuellos de botella derivados del crecimiento a través del fortalecimiento de recursos humanos e institucionales mediante el convenio con universidades como la Universidad Nacional del Noroeste de la Provincia de Buenos Aires (UNNOBA); 2) sostener las ventajas naturales mediante el cuidados del ambiente y prácticas sostenibles, con buenas prácticas en el uso del agua y uso de energías alternativas y renovables; 3) consolidar la cultura de la calidad de la semilla; y 4) fortalecer la innovación, a través de proveedores de tecnología especializada y el observatorio para la semilla forrajera.

En el marco de estas líneas de acción, el Programa de Servicios Agrícolas Provinciales (PROSAP) lanzó políticas de financiamiento a la inversión que preveían un reintegro de hasta el $40 \%$ de inversiones en compra de equipos de riego, perforaciones y pozos, y todo lo referente al manejo del agua ${ }^{(46)}$. Este tipo de incorporación tecnológica, que mejoran la eficiencia agronómica en el manejo del agua -por ejemplo, del riego por aspersión comparado con el riego gravitacional- se relaciona tanto con el segundo objetivo, sobre la sustentabilidad, como el tercero, vinculado a la calidad de la semilla a obtener. Por ello el riego y el manejo de los recursos hídricos subterráneos es uno de los ejes estratégicos del Plan de Mejora Competitiva y una problemática recurrente en lo que refiere al clúster.

En este sentido, uno de los proyectos del Plan de Mejora Competitiva es el denominado "proyecto riego" que busca mejorar el manejo del agua a través de la elaboración de una guía de "buenas prácticas", que son genéricamente definidas como "las acciones involucradas en la producción, procesamiento y transporte de productos de origen agropecuario, orientadas a asegurar la inocuidad del producto, la protección del medio ambiente y del personal que trabaja en la explotación"(47). En el marco de esta política también se están realizando estudios sobre la capacidad de explotación de los recursos hídricos subterráneos y la calidad del agua. 
Así, uno de los miembros del directorio del clúster, al presentar los resultados de los proyectos en 2014, destacaba: "Hoy es viable tener una red de monitoreo de los pozos que bombean la región y esto nos permitiría administrar mejor los recursos hídricos"(48).

Pero más allá de lo referente específicamente a la cuestión hídrica, lo que los funcionarios destacan de esta política pública es que el clúster es un "modelo", explícitamente: "un modelo de trabajo en el que actores del sector público y privado están presentes para potenciar sus capacidades y dar impulso a una temática tan importante ${ }^{\prime \prime(48)}$. En la misma línea, el entonces Ministro de Agricultura Julián Domínguez, al momento de la firma del convenio de cooperación en 2009, afirmaba:

El emprendimiento al que asistimos hoy, se enmarca dentro de la Nación que queremos. Ya no queremos venderle al mundo materias primas, sino que, queremos poder ofrecerle nuestra investigación, el desarrollo tecnológico, el conocimiento científico, la formación de nuestros cuadros técnicos y, sobre todo, la capacidad asociativa que tienen nuestros emprendedores privados. ${ }^{(22)}$

Siguiendo con este mandato, en 2018 se constituyó la ONG "Cluster de la semilla" bajo el formato de asociación civil con el objetivo de acceder a financiación de mayor envergadura destinada a proyectos público-privados ${ }^{(49)}$.

Del mismo modo, como política de desarrollo aplicada a la producción de semillas, es significativa la declaración de un funcionario -presidente del Instituto Nacional de Semillas- en el video institucional del clúster, disponible en el sitio web oficial, donde afirma que "la semilla es el vehículo de la tecnología". Esto se asocia a la concepción que se tiene del Estado y a la función que se espera que este desempeñe en materia de desarrollo. Como afirma otro de los funcionarios de dicho organismo en una ponencia presentada en un Congreso Nacional de Innovación en el Estado, con relación al clúster: [se] proyecta al Estado como un actor activo en el desarrollo de políticas públicas innovadoras, colaborando con instituciones flexibles y adecuadas a las necesidades de los operadores [...] cumpliendo el Estado innovador un rol catalizador y estimulador, que lidera el proceso de desarrollo económico y la construcción de un ambiente de participación y cooperación público-privado. ${ }^{(50)}$

La semilla, es un objeto híbrido por excelencia: natural (reservorio genético de la vida vegetal) y social (producto de la manipulación humana y del saber técnico). Al mismo tiempo es un actor que transporta conocimiento y articula a su alrededor todo un sistema de producción que integra el agua y la tecnología de riego como piezas claves de un ensamblaje que se visibiliza en la construcción del clúster, tanto territorial como discursivamente. En este ensamblaje, el Estado se posiciona como facilitador del acceso al capital para realizar la inversión a través de líneas de financiamiento específicas, y aportando conocimiento experto a partir del manual de las buenas prácticas. Esta acción tiene el efecto de legitimar y autorizar la acción de los agentes privados, promoviendo acciones que se adecuen explícitamente a sus intereses. Todo ello en función de un ideal de país al que se aspira: competitivo y que genere riquezas por la venta de más productos en el mercado mundial. Para alcanzarlo, se propone instrumentalmente la cooperación público-privada que conlleva un determinado modelo de Estado, portador de una moral del desarrollo y que al mejor estilo durkheimiano, es un órgano del pensamiento social, un actor racional que fija objetivos y arbitra medios institucionales para alcanzarlos. Pero este modelo, no es exclusivo del Plan de Mejora Competitiva como veremos a continuación.

\section{Plan Nacional de Riego}

El Plan Nacional de Riego es una política nacional del Ministerio de Agroindustria que busca duplicar la superficie bajo riego del país, 
pasando de 2,1 millones de ha que se riegan, según las últimas cifras disponibles de 2015, a 4,2 millones para 2030. En un comunicado oficial se anuncia que el Plan consiste en:

...formular un paquete de políticas públicas encaminadas a promover la expansión del riego y su eficiente utilización, creando una instancia institucional, a nivel nacional, que se hiciera cargo de definir y ejecutar estas políticas y que coordinara los esfuerzos existentes que desde hace tantos años se vienen desarrollando desde el PROSAP, técnicos de diversas instituciones de investigación y gobiernos provinciales. ${ }^{(51)}$

El Plan Nacional de Riego está articulado con una serie de programas pensados para lograr dicho aumento de la superficie bajo riego. El más relevante para nuestro trabajo en el norte de la provincia de Buenos Aires es la expansión del riego mecanizado con uso de agua subterránea. Para ello se intenta facilitar la inversión en equipos de riego a través de un financiamiento del Banco de la Nación Argentina con una tasa subsidiada. También se prevé la realización de estudios de exploración y monitoreo del agua subterránea. Para este eje destinará U\$1.600 millones de dólares, de un presupuesto total de U\$6.500 millones de dólares.

En mayo de 2018, el Ministerio consolidó el Plan Nacional de Riego mediante la Resolución 108/2018, y redobló la apuesta proponiendo triplicar la superficie bajo riego para llegar a 6 millones de ha para 2030. En su Artículo $2^{\circ}$ sostiene la necesidad de:

...ampliar la disponibilidad de recursos financieros, tanto públicos como privados, para la promoción de inversión privada en modernización o expansión de la superficie bajo riego, a través de la conformación de Asociaciones PúblicoPrivadas, negociación entre el Ministerio de Agroindustria con bancos nacionales y provinciales de las líneas de crédito disponibles y otras a crearse con tasas de interés promocionales". ${ }^{(52)}$
El programa será coordinado por una "Mesa Nacional de Riego" integrada por representantes de "instituciones del gobierno nacional, de gobiernos provinciales, de autoridades provinciales del agua y de organizaciones de usuarios, entre otros". Como antecedente, una mesa de competitividad de regantes se reunió por primera vez en ese mismo mes, en la que discutieron las principales limitaciones para la expansión del riego mecanizado: el costo de la energía y la falta de financiamiento adecuado para una inversión a largo plazo.

Sin embargo, más allá de estas iniciativas gubernamentales puntuales para la expansión del riego complementario, el objetivo más ambicioso del Plan Nacional de Riego es sumar inversión pública y privada a proyectos de riego para áreas extrapampeanas, según lo manifiesta la resolución antes citada. Esta política se propone desarrollar el potencial de irrigación bajo esquemas de asociación público-privada entre el gobierno nacional, las provincias y los productores usuarios del agua, denominadas "Corporaciones de Riego", al estilo de la Corporación Puerto Madero, una empresa sociedad anónima de propiedad estatal creada en 1989 para urbanizar 170 ha del antiguo puerto de Buenos Aires.

Las asociaciones público-privadas tienen un plazo de operación, que se ubicaría entre 50 y 70 años, un plan de inversiones de 4 a 6 años y de devoluciones en concepto de recupero a 12 o 15 años $^{(53)}$. Para lograr esas asociaciones, el Banco Mundial y la FAO enviaron a un equipo de expertos internacionales a fin de delinear el marco normativo para las uniones ${ }^{(54)}$.

Cada corporación tendría un órgano de gobierno integrado por el gobierno nacional, los gobiernos provinciales, los productores y los inversores. Cada uno realiza un aporte a la política de desarrollo: el gobierno nacional es quien promueve el plan y eventualmente realiza parte de la inversión en infraestructura o provee las garantías para la inversión; los gobiernos provinciales administran el agua, proporcionando cupos para su uso y son coinversores en el desarrollo de las 
corporaciones por la reinversión de los incrementos de los impuestos, como al valor de la tierra; los productores son los usuarios del agua y los propietarios de las tierras; $y$, finalmente, los inversores, aportan el capital para la adquisición de tecnología y construcción de infraestructura, en forma de pequeñas represas, acueductos, estaciones de bombeo y también otras obras complementarias, ya sea de índole eléctrica o logística.

Para las inversiones, se prevén fideicomisos que reciben capitales nacionales, pero sobre todo los ideólogos de esta política esperan inversiones extranjeras directas "aprovechando el retorno a los mercados internacionales y a la imagen positiva que tiene la Argentina como productora de alimentos" (según manifestaba uno de los funcionarios del ministerio a cargo de la cartera de la que depende el Plan Nacional de Riego ${ }^{(54)}$. Estos fideicomisos conllevan una estructura financiera compleja que tiene como garantía la venta y apropiación no solo del agua, sino de la tierra. Tal como fue publicado por el diario La Nación, el 27 de agosto de 2016, donde se explicaba el mecanismo:

Los fideicomisos van a poder emitir bonos, que van a ser el vehículo por el cual se repague la inversión en base a la venta parcial de la propiedad privada de los productores en la corporación. En rigor, la idea es que exista para cada región una masa crítica de productores interesados en participar en las corporaciones y en firmar una opción de venta futura de su campo cuando tienen el agua y la energía en el establecimiento. Las obras van a ser de cuatro a seis años contra la cesión futura de la tierra para permitir el repago precisamente de la inversión. En este sentido, una vez que se sustancia la obra un porcentaje de la tierra pasa a dominio efectivo del fideicomiso y eso va a permitir la reventa de un porcentaje de la tierra. Se crea así un valor de cambio inmobiliario que financia la infraestructura. Por lo pronto, ya se está avanzando en las primeras carpetas de inversiones y proyectos con Río Negro, Neuquén y
Chubut, provincias donde está el 40\% del potencial inicial de las más de 835.000 hectáreas definidas. ${ }^{(54)}$

En esta política vuelve a observarse el rol del Estado como organizador del proceso de acumulación, articulando entre los privados y desarrollando medidas en favor de sus intereses. Pone de relieve cómo, si bien el agua es el recurso que valoriza la tierra, la propiedad de la tierra es el factor clave para la apropiación de renta, mientras que la apropiación/ expropiación del agua aparece encubierta en el diseño de la política. La falta de regulación efectiva del uso del recurso es evidente cuando los permisos de riego no se realizan sobre el conocimiento de las fuentes de las fuentes de agua subterráneas. La contraprestación que realizarían los regantes por el uso del recurso permanece incierta, dando lugar a una apropiación no controlada, como se ve claramente en la siguiente de las políticas analizadas.

\section{La gestión del agua subterránea para el riego productivo}

Por último, analizo la política de gestión del agua subterránea para riego productivo que está contenida en un corpus normativo que regula la actividad en la provincia de Buenos Aires. Siendo un recurso común, vital y finito, el agua en Argentina es considerada un bien público de propiedad estatal(55). La potestad de su administración está a cargo de los Estados provinciales. Para ello, las provincias regulan los usos y aprovechamientos del recurso a través de un Código de Aguas. En el caso de la provincia de Buenos Aires este código está formulado en la Ley 12257, promulgada en 1999, la cual establece como autoridad de aplicación de la ley a la Autoridad del Agua, una entidad dependiente del Poder Ejecutivo Provincial que es la encargada de "la planificación, el registro, la constitución y la protección de los derechos, la policía y el cumplimiento y ejecución de las demás misiones que emanan del Código" (Artículo 3) ${ }^{(56)}$.

En el caso del agua subterránea para riego, su explotación requiere formalmente un per- 
miso por parte de la Autoridad del Agua. Para obtenerlo, los usuarios deben realizar un trámite ante el organismo que incluye presentar un informe agronómico firmado por un ingeniero y un informe de la calidad constructiva de la perforación, avalado por un perforista habilitado. Según los entrevistados, por falta de adecuación a la norma escrita, los trámites para pedidos de autorización -que tienen una vigencia de 4 años-, se demoran años en recibir una respuesta y terminan siendo frecuentemente rechazados (notas del trabajo de campo, septiembre 2016).

Estas dilaciones atentan contra los planes de los usuarios que tienen créditos para hacer la inversión en el equipo de riego y contratos de producción que cumplir en el corto plazo. En el caso de la producción de semillas, este plazo puede ser de apenas un mes. Según los usuarios, la legislación de la Autoridad del Agua para otorgamiento de permisos de agua subterránea es "impracticable". Eso hace que la amplia mayoría de las perforaciones en la provincia de Buenos Aires estén en condición de "ilegalidad". A pesar de ser una situación ampliamente conocida y reconocida, la Autoridad del Agua tampoco controla ni ejerce su poder de policía. Por eso desde el punto de vista práctico, no hay regulación de hecho y el régimen de explotación es abierto.

Dejar de regar por falta de autorización formal no es una opción para las empresas semilleras o para los productores. Así, las perforaciones se hacen "lo más prolija que puedas", siguiendo los requerimientos que estipula la ley en términos de impacto ambiental, calidad constructiva de los pozos, etc., y se las declara como preexistente para intentar regularizarla después cuando ya se encuentran funcionando, es decir, "post facto". Eso hace que las perforaciones sean "clandestinas", porque "no tienen permiso de explotación, no tiene papeles, no tiene nada" (Encargado de producción, Pergamino, mayo 2017).

Al mismo tiempo, no existen aún trabajos integrales sobre la dinámica del agua subterránea con miras a su regulación, siendo que la preocupación por la calidad de dicho recurso es un problema importante asociado al nivel de las extracciones ${ }^{(9)}$. La falta de conocimiento sobre las características del acuífero y su dinámica hacen también que las pretensiones de regulación existan solo a nivel formal, en la ley escrita y en los proyectos de desarrollo.

Saliendo del pequeño círculo de ingenieros que trabajan en la industria semillera, los productores no tienen claro conocimiento acerca del procedimiento o las obligaciones que implica tener un pozo de explotación de agua para riego. Al estar al servicio de la producción de semillas, las empresas son las que se ocupan de los aspectos formales relacionados con las perforaciones. Con la tendencia a la tercerización y la proliferación de los convenios de producción, los productores tuvieron que comenzar a ocuparse mayormente de estas cuestiones, pero hasta el momento del trabajo de campo (año 2017) no era una problemática ampliamente reconocida. Esto resultaba evidente en el hecho de que incluso muchos regantes ni siquiera conocían la existencia de la Autoridad del Agua o el nombre del organismo público encargado de la regulación del uso que ellos hacían del agua subterránea.

De cualquier modo, la omisión del Estado en esta materia ha generado intranquilidad en ciertos usuarios por la irregularidad en la que se encuentran sus perforaciones. Esta situación los deja en una posición de vulnerabilidad ante un eventual conflicto por el uso del recurso, que puede terminar judicializado.

Además, tanto los usuarios como los proveedores de tecnología de riego temen restricciones a la explotación del recurso que pongan en peligro el sistema de producción bajo riego complementario y el negocio de la producción de semillas híbridas. Por eso, ante la falta de soluciones desde la Autoridad del Agua, las empresas semilleras, por un lado, y los productores regantes, por el otro, instrumentaron, desde sus propias organizaciones, mecanismos para legitimar el uso que hacen del agua.

A través de la Asociación de Semilleros Argentinos, en el caso de los semilleros, o de la Asociación de Riego Pampeano (ARP), 
organización de carácter gremial que reúne cerca de 1.300 de regantes de la provincia de Buenos de Aires, los usuarios del agua subterránea tributan al Estado un canon por derecho de uso -según la Resolución $465 / 2013^{(57)}$-, aunque las perforaciones sean "clandestinas" y no estén regularizadas. Lo Ilamativo es que según a través de qué asociación se canalice el pago, su forma de cálculo varía, poniendo en evidencia su carácter ad hoc. Es decir, cada organización tiene un convenio particular con la Autoridad del Agua a través del cual se estipula el canon que paga el usuario por el derecho a uso, sin que ello implique la autorización de la perforación. En el caso de la Asociación de Semilleros Argentinos acordó un monto fijo por perforación utilizada que asciende a $\$ 5.000$ por campaña -en teoría actualizable periódicamente según el Decreto 427/13 $3^{(58)}$ - que se paga desde $2014^{(59)}$ y que se encontraba vigente al momento del trabajo de campo (noviembre de 2017). Mientras que, por otro lado, la Asociación de Riego Pampeano había acordado en 2011 un monto calculado sobre el precio del gasoil. Como dice la Resolución, se espera el "aporte anual a esta Repartición, en pesos equivalente a doscientos litros (200 lts.) de gasoil por cada pozo activo que tenga documentación visada por la Autoridad del Agua, dentro del plazo perentorio de sesenta (60) días, para el Ordenamiento del Riego Productivo" ${ }^{\prime(60)}$.

La aceptación por parte del Estado de este "arreglo" pone en evidencia lo que los entrevistados sostienen en reiteradas ocasiones: que incluso los funcionarios del propio organismo admiten la falta de adecuación de las normas para la autorización de los pozos. Este hecho ha sido públicamente reconocido por el mismo organismo en la Resolución 333/17 en la cual establece en los "considerando" que:

...desde 2003, fecha en que se creó el organismo hasta la actualidad, esta Autoridad del Agua se ha regido por distintas normativas con sendos procedimientos y requisitos para el otorgamiento de las autorizaciones y/o permisos mencionados que no han tenido el impacto deseado;
Que dicha conclusión se obtiene por un lado de la observación de la muy baja o prácticamente nula cantidad de Usuarios del Recurso Hídrico que se encuentran debidamente empadronados o registrados como permisionarios o concesionarios y, por otro lado, de la existencia de más de 15.000 expedientes correspondientes a 8.500 usuarios aproximadamente, con tramitaciones inconclusas que tienen un promedio de 5 años de demora en la evaluación por causa de distinta índole. ${ }^{(61)}$

En este contexto, y ante la imposibilidad de tener las perforaciones en regla, pagar canon se convirtió en el "salvoconducto" para la industria semillera y los regantes. Desde 2013, el pago del canon implica registrarse en el Banco Único de Usuarios de Recursos Hídricos (BUDURH) ${ }^{(57)}$ y declarar la ubicación de la perforación, los niveles dinámicos y estáticos del agua subterránea, la cantidad extraída y la presentación de un balance hídrico por campaña -cuánto Ilovió y cuánto se regó-, entre otra información requerida en las declaraciones juradas y atestiguada en la documentación probatoria exigida ${ }^{(61)}$. De esta manera, la Autoridad del Agua empieza a componer un padrón de usuarios y un registro de las perforaciones activas en cada campaña que, aunque está lejos de ser sistemático y exhaustivo, representa en sí mismo un avance significativo hacia la regulación.

En 2017, la Autoridad del Agua estableció una reforma en el reglamento de inscripción, autorización y otorgamiento de permisos de explotación de agua que consta en la Resolución 333 de ese año, en la que se da cuenta de la reingeniería del procedimiento para la obtención de permisos que implica simplificar los trámites, bajar tasas, digitalizar las presentaciones y autorizar gestores externos. Estos intentos de ordenamiento del uso del agua son vistos por los productores como razonables, aunque siempre temen la exigencia de nuevas erogaciones. Saben que lo próximo a exigir es un dispositivo medidor de caudales y, luego, el pago por caudal 
consumido. De cualquier manera, ante la falta de controles, la voluntad de declarar las perforaciones y cumplir con este trámite depende actualmente de cada usuario ${ }^{(21)}$.

Si bien en Buenos Aires la gestión del agua subterránea para riego productivo no la realizan los usuarios del recurso a través de una organización propia como ocurre por ejemplo en Córdoba -la segunda provincia con mayor cantidad riego mecanizado después de Buenos Aires- sí existe en la provincia la idea de avanzar hacia esa dirección. Ya desde 2016, cuando nuevas autoridades se hicieron cargo del organismo gubernamental bajo el gobierno de María Eugenia Vidal, surgió la iniciativa de reorganizar la gestión del agua subterránea pensando en alternativas a los Comité de Cuencas, dándole más participación al sector privado, delegando atribuciones de la Autoridad del Agua, bajo el formato de consorcios de usuarios (notas del trabajo de campo, agosto 2016). Estas ideas son ratificadas en la presentación del presupuesto 2018 de la Autoridad del Agua donde proponen "organizar y establecer consorcios de usuarios para riego productivo" (62). Sin embargo, hasta el momento, estas iniciativas no se han efectivizado en propuestas institucionales concretas.

Lo llamativo es que el "salvoconducto" a la propia incapacidad de regular el uso del recurso por parte de Estado también es una política del mismo Estado. Organizar consorcios de usuarios implicaría, de igual modo, delegar en el sector privado funciones públicas que hasta el momento no ha podido desempeñar el organismo de control del agua. En esta política, quizás más evidente aún que en las otras, la acción del Estado es acompañar los desarrollos existentes, buscando maneras de regularizar acciones que hasta el momento se desarrollaban por fuera de su control.

\section{DISCUSIÓN FINAL}

"¿Cómo es que la Argentina no logra desarrollarse? ¿Por qué los índices de crecimiento no son explosivos como los que esperamos de un país como este?" publica el diario Infobae a propósito de la conferencia de relanzamiento del Plan Nacional de Riego, con declaraciones de representantes de empresas trasnacionales, proveedoras de tecnología de riego. El artículo ofrece la siguiente explicación:

...la respuesta está en la infraestructura. "Es un factor determinante: electricidad, caminos, obras para encauzar el agua y mejorar los canales. Hay campos que tienen buenos suelos, condiciones climáticas ideales, los productores conocen cuáles son los mejores cultivos que mejor se desarrollan en la zona, pero muchas veces les falta agua, porque no llueve lo suficiente." ${ }^{\prime(63)}$

Sobre la base de estas ideas se construye la noción de que la expansión del riego es una cuestión de desarrollo, incluso para las zonas húmedas del país, y las tres políticas públicas a las que hicimos referencia apuntan en ese sentido. El Plan de Mejora Competitiva es una política regional participativa, el Plan Nacional de Riego es una política nacional en cuyo diseño intervienen consultores internacionales, y la gestión de agua subterránea es una política provincial con marcado carácter burocrático, llevada a cabo por funcionarios que administran procedimientos desde oficinas radicadas en la capital provincial, con escasa o nula intervención en el territorio. Pero más allá de estas diferencias, analizadas en conjunto muestran una coherencia que tiene que ver con la concepción de lo público y lo privado.

El ensamblaje que se genera entre los discursos de desarrollo, el agua, la tierra, las semillas, la tecnología mecánica, la biotecnología, los saberes expertos, los actores privados, las empresas transnacionales y el Estado dan lugar a la emergencia de la tierra irrigada. En este paisaje hídrico el agua queda bajo control de los actores capitalistas que pueden hacer pago de la inversión en riego, mientras el Estado idea medios institucionales para legitimar la sesión del recurso y autorizar su uso demandando contrapartidas mínimas, en el mejor de los casos. 
Las políticas presentadas exhiben una concepción particular del Estado: un poder legitimador, más que regulador, que entiende que son los actores privados actuando en el mercado los que generan la riqueza y, por lo tanto, tienen derecho de apropiársela. En esta transformación, el capital global tiene una participación importante, reteniendo parte de los beneficios de la explotación del agua. Las semilleras transnacionales propietarias de las licencias biotecnológicas, al igual que las principales proveedoras de equipos y los inversores en proyectos de riego son actores claves en el impulso de estos desarrollos.

En ellos, la tierra es el recurso visible, el que se compra, se alquila, o con el que se paga, mientras que el agua aparece invisibilizada. La "tierra irrigada" es la conjunción de ambos, cada uno sujeto a su propio régimen de propiedad: el primero, como propiedad privada y, el segundo, como propiedad estatal, es decir, público, pero que en su asociación se resignifican. Es la propiedad de la tierra la que abre el camino a la privatización del agua en su apropiación como mercancía. Por lo tanto, la mercantilización del agua es un efecto oculto de este ensamblaje, pero que adquiere un carácter evidente cuando los actores tienen necesidad de cuantificar la extracción del recurso asignándole un valor monetario al milímetro regado.

En este trabajo, presenté una serie de ideas sobre las políticas públicas y el rol del Estado en la agricultura bajo riego que servirán de guía para profundizar en la comprensión del desarrollo de este capitalismo agrario. A partir de la revisión de estas políticas procuré reflexionar sobre preguntas como ¿qué es lo que está en juego con la creación de la tierra irrigada?, ¿cuál es el rol de lo público y cuál el de lo privado?, ¿quién usa el agua?, ¿quién se apropia de su renta? A partir de estos interrogantes y entendiendo que las políticas públicas contienen modelos implícitos y explícitos de sociedad y operan como herramientas para imponer orden y coherencia, se observa que las políticas revisadas intentan aproximar las normas escritas a las formas de hacer sancionadas desde la práctica, es decir a un forma de hacer agricultura que controla técnicamente el agua en el proceso de producción y, al hacerlo, pone en marcha un proceso social de apropiación, acumulación y legitimación.

\section{AGRADECIMIENTOS}

A la Universidad de Buenos Aires y al Consejo Nacional de Investigaciones Científicas y Técnicas. A Claudia Guebel por invitarme a participar de la mesa sobre políticas públicas y espacio en las Jornadas de Antropología Social, Facultad de Filosofía y Letras, Universidad de Buenos Aires, que sirvió de inspiración para escribir este artículo. A Néstor Barrionuevo del Instituto Nacional de Tecnología Agropecuaria (INTA), por compartir conmigo su valioso trabajo en teledetección del riego, a Alejandro Gaggero por sus lecturas y comentarios sobre versiones anteriores de este texto, y a los revisores anónimos de la revista por sus sugerencias y recomendaciones. Como autora, soy única responsable de las ideas aquí expresadas.

\section{REFERENCIAS BIBLIOGRÁFICAS}

1. Villholth KG, Lopez-Gunn E, Conti K, Garrido A, Van Der Gun J. Advances in groundwater governance. London: CRC Press; 2018. 
2. Kepfield SS. The "liquid gold" rush: Groundwater irrigation and law in Nebraska, 1900-93. Great Plains Quarterly. 1993;13(4):237-250.

3. Hoogesteger J, Wester P. Intensive groundwater use and (in)equity: Processes and governance challenges. Environmental Science \& Policy. 2015;51:117-124. doi: 10.1016/j.envsci.2015.04.004

4. Budds J. La demanda, evaluación y asignación del agua en el contexto de escasez: un análisis del ciclo hidrosocial del valle del río La Ligua, Chile. Revista de Geografía Norte Grande. 2012;52:167-184. doi: 10.4067/S0718-34022012000200010.

5. Riera C. Producción agrícola, tecnología y procesos de diferenciación social: vulnerabilidad e incertidumbre de los productores regantes de Córdoba. [Tesis Doctoral]. Buenos Aires: Universidad de Buenos Aires; 2015.

6. Castro JE. Apuntes sobre el proceso de mercantilización del agua: Un examen de la privatización en perspectiva histórica. En: Espinoza S. Justicia ambiental y sustentabilidad hídrica. Cochabamba: CGIAB; 2009.

7. O'Connor J. The second contradiction of capitalism. En: Benton T, (ed.). The greening of Marxism. New York: Guilford Press; 1996. p. 197-221.

8. Shah T. Taming the anarchy: Groundwater governance in South Asia. Washington DC: Routledge; 2009.

9. Aeschbach-Hertig W, Gleeson T. Regional strategies for the accelerating global problem of groundwater depletion. Nature Geoscience. 2012;5(12):853-861.

10. Harvey D. El nuevo imperialismo. Madrid: Akal; 2004.

11. Marx K. La Ilamada acumulación originaria. En: El Capital. Tomo I. Buenos Aires: Siglo XXI Editores; 1983 [1867]. p. 891-954.

12. Swyngedouw E, Despojo y re-politización del agua: hacia una nueva política de los comunes. En: Arroyo A, Boelens R, (ed.). Aguas robadas: despojo hídrico y movilización social. Quito: Justicia Hídrica, IEP y Abya Yala; 2013. p. 11-16.

13. Ostrom E. Governing the commons. Cambridge: Cambridge University Press; 1990.

14. Ullberg de Baez S. Marcas de agua: Un análisis antropológico de inundaciones urbanas y memoria social en la ciudad de Santa Fe. En: Gustafsson M-T, Uggla F, (eds.). Pensamiento social sueco sobre América Latina. Buenos Aires: CLACSO; 2016. p. 267-296.

15. Oszlak O, O’Donnell G. Estado y políticas estatales en América Latina: Hacia una estrategia de investigación. Buenos Aires: CEDES; 1976. (Documento G. E. CLACSO $\left.N^{\circ} 4\right)$.

16. Shore C. La antropología y el estudio de la política pública: reflexiones sobre la "formulación" de las políticas. Antípoda. 2010;10:21-49.
17. Roth A. Políticas públicas: Formulación, implementación y evaluación. Bogotá: Ediciones Aurora; 2002.

18. Hastrup K. Water and the configuration of social worlds: An anthropological perspective. Journal of Water Resource and Protection. 2013;5(4):59-66. doi: 10.4236/jwarp.2013.54A009.

19. Boelens R. Water justice in Latin America: the politics of difference, equality, and indifference. Amsterdam: Center for Latin American Research and Documentation; 2015.

20. Krause F, Strang V. Thinking relationships through water. Society \& Natural Resources. 2016;29(6):633-638.

21. Linton J, Budds J. The hydrosocial cycle: Defining and mobilizing a relational-dialectical approach to water. Geoforum. 2013;57:170-180. doi: 10.1016/j.geoforum.2013.10.008.

22. Swyngedouw E. The political economy and political ecology of the hydrosocial cycle. Journal of Contemporary Water Research \& Education. 2009;142:56-60. doi: 10.1111/j.1936-704X.2009.00054.x.

23. Escobar A. La invención del Tercer Mundo: construcción y deconstrucción del desarrollo. Santafé de Bogotá: Editorial Norma; 1998.

24. Zwarteveen M, Boelens R. La investigación interdiciplinaria referente a la temática de "justicia hídrica": unas aproximaciones conceptuales. En: Boelens R, Cremers L, Zwarteveen M, (eds.). Justicia hídrica: acumulación, conflicto y acción social. Lima: IEP, Fondo Editorial PUCP; 2011. p. 29-59.

25. Budds J. Relaciones sociales de poder y la producción de paisajes hídricos. En: Boelens R, Cremers L, Zwarteveen M, (eds.). Justicia hídrica: acumulación conflicto y acción social. 15. Lima: IEP, Fondo Editorial PUC; 2011. p. 59-70.

26. Mukherji A, Shah T. Groundwater socio-ecology and governance: a review of institutions and policies in selected countries. Hydrogeology Journal. 2005;13:328345.

27. Sandoval R. A participatory approach to integrated aquifer management: the case of Guanajuato State, Mexico. Hydrogeology Journal. 2004;12(1):6-13.

28. Villar PC. As águas subterrâneas e o direito à água em um contexto de crise. Ambiente \& Sociedade. 2016;19(1):83-102. doi: 10.1590/1809-4422asoc1501 $26 r 1 v 1912016$.

29. López-Gunn E, Rica M. Gestión colectiva del agua subterránea en España. Santander: Fundación Botín; 2013.

30. Budds J. Contested H2O: Science, policy and politics in water resources management in Chile. Geoforum. 2009;40(3):418-430.

31. Kemper KE. Instruments and institutions for groundwater management. En: Giordano M, Villholth K. The agri- 
cultural groundwater revolution: Opportunities and threats to development. Colombo: CAB; 2007. p. 153-172.

32. Peck JC. Groundwater management in the High Plains Aquifer in the USA: Legal problems and innovations. En: Giordano M, Villholth K. The agricultural groundwater revolution: Opportunities and threats to development. Colombo: CAB; 2007. p. 296-319.

33. Steward DR, Bruss PJ, Yang X, Staggenborg SA, Welch SM, Apley MD. Tapping unsustainable groundwater stores for agricultural production in the High Plains Aquifer of Kansas, projections to 2110 . Proceedings of the National Academy of Sciences. 2013;110(37):e3477-e3486.

34. Zhang L, Wang J, Huang J, Rozelle S. Development of groundwater markets in China: a glimpse into progress to date. World Development. 2008;36(4):706-726.

35. Strang V. Infrastructural relations: Water, political power and the rise of a new 'despotic regime'. Water Alternatives. 2016;9(2):292-318.

36. Swyngedouw E. Social power and the urbanization of water: flows of power. Oxford: Oxford University Press; 2004

37. Latour B. Reassembling the social: an introduction to actor-network theory. Oxford: Oxford University Press; 2005.

38. Callon M. Is science a public Good? Science, Technology and Human Values. 1994;19(4):395-424. doi: 10.1177/016224399401900401.

39. Gezon L. Khat commodity chains in Madagascar: multi-sited ethnography at multiple scales. En: Vaccaro I, Smith EA, Aswani S, (eds.). Environmental social sciences: methods and research design. Cambridge: Cambridge University Press; 2010. p. 238-265.

40. Maxwell J. Qualitative research design: An interactive approach. London: Sage Publications; 1996.

41. Guber R. La etnografía: Método, campo y reflexividad. Buenos Aires: Norma; 2001.

42. Geertz C. Conocimiento local: Ensayos sobre la interpretación de las culturas. Barcelona: Paidós; 1994.

43. Programa de Servicios Agrícolas Provinciales. Plan de Mejora Competitiva: Cluster de la Semilla. Buenos Aires: PROSAP, UCAR, Ministerio de Agricultura, Ganadería y Pesca; 2013.

44. Romero FG. El Imperialismo y el agro argentino. Buenos Aires: CICCUS; 2016

45. Porter M. Los clusters y la competencia. Revista Gestión. 1999;1(2):130-145

46. La Opinión. Impulso a la inversión para el sector agropecuario a través del Prosap. La Opinión [Internet]. 7 feb 2016 [citado 10 mar 2018]. Disponible en: https:// tinyurl.com/y8jq9v8r.
47. Instituto Nacional de Tecnología Agropecuaria. Acerca de la Buenas Prácticas Agrícolas [Internet]. 21 jun 2016 [citado 10 mar 2018]. Disponible en: https://tinyurl.com/yaz5zgp4.

48. Democracia. Resultados de proyectos del Clúster de la Semilla. Democracia [Internet]. 7 may 2014 [citado 15 oct 2018]. Disponible en: https://tinyurl.com/y75u6egz.

49. Partido de Pergamino, Dirección de Comunicación y Prensa. Se conformó la Asociación Civil del Cluster de la Semilla [Internet]. 13 abr 2018 [citado 10 oct 2018]. Disponible en: https://tinyurl.com/ycz6op56.

50. Gregorio L. Un Estado innovador que absorbe conocimiento y lo invierte en desarrollo regional. Congreso Nacional de Innovación en el Estado (CONIE); Resistencia, Chaco, 2015.

51. Ministerio de Agricultura, Ganadería y Pesca. El Plan Nacional de Riego: la continuidad de las políticas públicas orientadas al sector. Riegos y Drenajes. 2015;(75):48.

52. Ministerio de Agroindustria. Resolución 108/2018: Plan Nacional de Riego [Internet]. 23 may 2018 [citado 15 oct 2018]. Disponible en: https://tinyurl.com/ya9mjvw5.

53. López P. El Gobierno lanza plan para incorporar un millón de hectáreas a sistema de riego. El Cronista [Internet]. 23 may 2016 [citado 15 oct 2018]. Disponible en: https://tinyurl.com/y9dcbdnf.

54. Bertello F. Cómo el riego puede aportar inversiones por US\$ 4254 millones. La Nación [Internet]. 27 ago 2016 [citado 10 oct 2018]. Disponible en: https://tinyurl. com/yctrooqz.

55. Riera C. La tecnología de riego y la disputa por el agua subterránea en Córdoba, Argentina. Caderno de Geografía. 2017;27(48):27-43. doi: 10.5752/p.2318-2962.2017 v27n48p27.

56. Usunoff E, Peluso F, Castelain JG, Miranda M. Hacia la gestión integrada de los recursos hídricos en la provincia de Buenos Aires, Argentina. Águas Subterrâneas. 2000;(Supl):S1-S21.

57. Gobierno de la Provincia de Buenos Aires, Autoridad del Agua. Resolución 465/2013 [Internet]. 17 oct 2013 [citado 10 oct 2018]. Disponible en: https://tinyurl. com/y8cyufhd.

58. Gobierno de la Provincia de Buenos Aires, Autoridad del Agua. Resolución 257/2014 [Internet]. 29 abr 2014 [citado 10 oct 2018]. Disponible en: https://tinyurl. com/ydeeasmr.

59. Gobierno de la Provincia de Buenos Aires, Autoridad del Agua. Resolución 001. Buenos Aires: ADA; 2013.

60. Gobierno de la Provincia de Buenos Aires, Autoridad del Agua. Resolución 135. Buenos Aires: ADA; 2011.

61. Gobierno de la Provincia de Buenos Aires, Autoridad del Agua. Resolución 333/2017 [Internet]. 2 may 2017 [citado 10 oct 2018]. Disponible en: https://tinyurl. com/yd6rezzw. 
62. Provincia de Buenos Aires, Dirección Provincial de Presupuesto Público. Presupuesto General Ejercicio 2018: Autoridad del Agua [Internet]. 2018 [citado 12 dic 2018]. Disponible en: https://tinyurl.com/ya7fsk2e.
63. Infobae. Está en marcha el Plan Nacional de Riego para 6 millones de hectáreas. Infobae [Internet]. 3 jun 2018 [citado 12 dic 2018]. Disponible en: https://tinyurl. com/y7ngp3bb.

\section{FORMA DE CITAR}

Riera C. La "tierra irrigada" y la mercantilización del agua en un nuevo paisaje hídrico de la agricultura pampeana: el caso del clúster de la semilla. Salud Colectiva. 2020;16:e2325. doi: 10.18294/sc.2020.2325.

Recibido: 24 may 2019 | Versión final 5 may 2020 | Aprobado: 17 may 2020 | Publicado en línea: 21 jul 2020

Esta obra está bajo una licencia de Creative Commons Reconocimiento-NoComercial 4.0

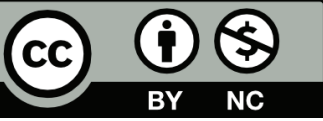
Internacional. Reconocimiento - Permite copiar, distribuir y comunicar públicamente la obra. A cambio, se debe reconocer y citar al autor original. No Comercial - Esta obra no puede ser utilizada con finalidades comerciales, a menos que se obtenga el permiso. 